\title{
Geometric nonlinearities in field theory, condensed matter and analytical mechanics
}

\author{
J.J. Sławianowski \\ Institute of Fundamental Technological Research, Polish Academy of Sciences, \\ $5^{B}$ Pawińskiego Str., 02-106 Warsaw, Poland
}

Received October 05, 2010, in final form October 12, 2010

There are two very important subjects in physics: Symmetry of dynamical models and nonlinearity. All really fundamental models are invariant under some particular symmetry groups. There is also no true physics, no our Universe and life at all, without nonlinearity. Particularly interesting are essential, non-perturbative nonlinearities which are not described by correction terms imposed on some well-defined linear background. Our idea in this paper is that there exists some mysterious, still incomprehensible link between essential, physically relevant nonlinearity and dynamical symmetry, first of all, of large symmetry groups. In some sense the problem is known even in soliton theory, where the essential nonlinearity is often accompanied by the infinite system of integrals of motion, thus, by infinite-dimensional symmetry groups. Here we discuss some more familiar problems from the realm of field theory, condensed matter physics, and analytical mechanics, where the link between essential nonlinearity and high symmetry is obvious, although not fully understandable.

Key words: Born-Infeld electrodynamics, condensed matter, general relativity and tetrads, non-perturbative nonlinearity, relativistic structured continuum, dynamical symmetry

PACS: 11.30.-j, 05.45.-a, 46.05.+b, 04.20.-q, 03.50.De, 45.20.Jj

\section{Symmetry versus nonlinearity in metrical and tetrad gravitation. Com- parison with mechanical toy models}

Something close to the Anthropic Principle and similar ideas:

There is no Our Cosmos and no Life without Nonlinearity.

In various aspects, quite trivial, every-day-life ones, and very fundamental structural problems. Concerning the every-day life, e.g., there is no thermal expansion of bodies without nonlinearity expressed by non-symmetric shape of diagram of the interparticle potential energy as a function of distance. Biological and ecological systems are based on the limit cycles impossible without nonlinearity. Similarly, without nonlinearity, at least in some background, it is impossible to reconcile the field equations and equations of motion, e.g., in Maxwell electrodynamics. Without nonlinearity there is no stochastization, no equipartition of energy and thus no thermodynamics. It is instructive to think of some crazy model of condensed matter as a system of mutually coupled harmonic oscillators, based, e.g., on the isotropic Lagrangians of the form:

$$
L=\frac{1}{2} \sum_{A} m_{A} \frac{\mathrm{d} x^{i} A}{\mathrm{~d} t} \frac{\mathrm{d} x^{j} A}{\mathrm{~d} t} g_{i j}-\frac{1}{2} \sum_{A \neq B} \varkappa_{A B}\left(x_{A}^{i}-x_{B}^{i}\right)\left(x^{j}{ }_{A}-x^{j}{ }_{B}\right) g_{i j},
$$

where $g$ denotes the metric tensor, $\varkappa_{A B}=\varkappa_{B A}$ are the elastic constants, and $m_{A}$ are the particle masses. The corresponding equations of motion have the form:

$$
m_{A} \frac{\mathrm{d}^{2}}{\mathrm{~d} t^{2}} x_{A}^{i}=-\sum_{B} \varkappa_{A B}\left(x_{A}^{i}-x_{B}^{i}\right) .
$$

Let us notice that the metric tensor does not enter (1.2) at all, although it is explicitly present in (1.1). This is one of its ambiguous roles in equations of physics. But, never mind, the point is that 
the model (1.1), (1.2) does prevent the decay of the system, but it does not prevent its collapse. The only anti-collapse means of (1.1), (1.2) is the centrifugal barrier. But this is physically nonsufficient, and the true anti-collapse repulsive potentials must be positively-singular at coincidences of particles; the corresponding forces will be certainly non-harmonic and the model essentially nonlinear. As harmonic models always split into mutually non-interacting one-dimensional normal modes, in quantum field theory, and classical field theory as its kindergarten, one accepts the view that the true interaction is encoded within the anharmonic sector. From this point of view the harmonic models are "non-interacting", although there exist some "springs" between non-normal modes. Finally, let us recall the immortal and in any case still unresolved problem of quantum decoherence. There is an "infinity" of ideas about it, one of them is some fundamental nonlinearity hidden somewhere beyond the usual pragmatic framework of linear quantum mechanics.

Nonlinearity is physically desirable, just unavoidable, but, at the same time, linear models are, in principle, explicitly treatable. This motivates a kind of compromise often dealt with in practice. Namely, one considers a certain linear background model with some extra imposed nonlinear perturbations. These perturbations are often considered to be "small", or to be more precise, they are controlled by some coupling parameter. The vanishing value of this parameter corresponds to the background linear model. To solve nonlinear problems, one employs certain perturbation techniques, expansions with respect to the "small" parameter, and the search of solutions in terms of asymptotic series (by collecting coefficients at the same power of the parameter). Of course, such a procedure is always more or less "tricky", certainly non-reliable. One never knows a priori if the underlying linear background is structurally stable under perturbations. And, what is more important, there exist fundamental theories and models which are essentially nonlinear. They are nonlinear from the very start and there is no natural splitting into linear background and nonlinear correction term. Let us mention general relativity, 'tHooft-Polyakov-Kleinert strings, Born-Infeld electrodynamics and its generalizations, Euler equation for ideal fluids which are all profound examples in fundamental field theory and in condensed matter physics. Incidentally, the two disciplines are not sharply distinct, and the border between them is rather diffused [1 8].

It very often happens in fundamental theories that the tensorial structure of considered objects just canonically induces certain coupling schemes and certain canonical nonlinearities. This is just the case with the mentioned examples, where one is faced with the peculiar convolution of two things: the essential non-perturbative nonlinearity and the huge symmetry groups. This convolution is in no way accidental and may be heuristically explained within the framework of variational theories. This is not an essential restriction, because usually, dissipative models presume a certain self-adjoint background, and besides, with certain modifications, the very idea works for them as well. Simply, it is quite easy to comprehend them using Lagrangian concepts. Namely, from the geometric point of view Lagrangian is a scalar W-density of weight one, built of dynamical variables, i.e., "fields", and their derivatives with respect to independent variables, let us say, "space-time" coordinates. In fundamental theories one deals in principle with first-order derivatives, but with certain delicate points concerning general relativity. However, to construct scalar densities or scalars from "fields", one usually needs certain "tools", which enable one to define invariant derivatives with respect to "space-time" coordinates, and to contract tensorial spatio-temporal or internal indices. In specially-relativistic or Galilean physics those tools are usually some metric tensors and their by-products such as affine connections, volume forms, etc. They are absolute objects of the theory. When they are kept fixed, symmetries of the theory are rather poor, because they should respect and preserve those objects. In linear theories the metric tensors enter Lagrangians via coefficients of quadratic forms built of dynamical quantities, e.g., in kinetic energy, in kinetical terms of field Lagrangians, etc. [8]. It also enters through covariant derivatives of fields, integration element, etc. When kept fixed as an absolute, controlling object, it restricts the symmetry group to the finite-dimensional isometry group of $g$. But it was just the general covariance idea of Hilbert that no absolute objects may exist in really fundamental theory [8]. If so, $g$ must be included into physical degrees of freedom and then the symmetry group of the theory becomes just Diff $M$, the group of all diffeomorphisms of the space-time manifold $M$. This is a huge, infinite-dimensional group. And automatically the theory becomes essentially nonlinear, 
without any linear background to be perturbed. And this is a rule: in linear variational theories the quadratic forms underlying Lagrangians, automatically restrict the symmetry group to some (pseudo-)Euclidean group. To escape this restriction, one must include the quadratic form itself into degrees of freedom, and this self-interaction brings about some essential nonlinearity [7, 8]. NonAbelian gauge theories provide another example of the relationship between essential nonlinearity and symmetry groups. The inherent nonlinearity and self-interaction of gauge fields (the "radiating radiation", so to speak) is exactly due to their symmetry group. Let us also mention the solitons, where one observes the very peculiar coincidence of the essential nonlinearity and the rich groups of hidden symmetries (hierarchy of constants of motion, the total intergrability). Generalized BornInfeld-type models (including 'tHooft-Polyakov-Kleinert) offer some very interesting mechanisms of essential nonlinearity, apparently without a direct link to symmetry [8 11]. However, a more detailed analysis shows that some important symmetries are also intimately connected with them.

It is very instructive to review the structure of nonlinearities quoted above, with the special stress on their geometric background, first of all, but not only, on symmetry groups. Certain common features of field theory, mechanics of continua, condensed matter theory, and analytical mechanics are then exhibited and the borders between them diffuse in a sense.

In general relativity, the Hilbert Lagrangian of the metric field $g$ on the space-time manifold $M$ is given by 12

$$
\mathcal{L}_{\mathrm{H}}[g]=\mathcal{L}_{\mathrm{H}}\left(g, \partial g, \partial^{2} g\right)=-\frac{1}{2 \varkappa} \mathcal{R}[g] \sqrt{|g|}
$$

with the obvious meaning of symbols: $\varkappa$ is proportional to the gravitation constant (the proportionality factor depends on the system of units), $\mathcal{R}[g]$ is the curvature scalar built of $g$, and $|g|$ is an abbreviation for the absolute value of $\operatorname{det}\left[g_{\mu \nu}\right]$ in a given coordinate system. Geometrically $|g|$ is a scalar $W$-density of weight two, and because of this, $\mathcal{L}_{\mathrm{H}}[g]$ is a scalar $W$-density of weight one, just as any correctly defined Lagrangian should be. Sometimes one modifies (1.3) by adding the cosmological term

$$
\mathcal{L}_{\operatorname{cosm}}[g]=\Lambda \sqrt{|g|}
$$

$\Lambda$ is here a constant parameter usually referred to as cosmological constant.

Some comments are necessary here. Namely, Lagrangian (1.3) depends on second derivatives, but the corresponding variational principle is essentially first-order one. The point is that $\mathcal{L}_{\mathrm{H}}$ quasilinearly depends on second derivatives, i.e., linearly with coefficients depending algebraically on $g$, but not on its first derivatives. The second derivatives in (1.3) may be absorbed into a total divergence term and removed from the action functional,

$$
\mathcal{L}_{\mathrm{H}}[g]=G_{\mathrm{H}}[g] \sqrt{|g|}+\text { "Div" }=G_{\mathrm{H}}(g, \partial g) \sqrt{|g|}+\text { "Div". }
$$

The first-order Lagrangian $G_{\mathrm{H}} \sqrt{|g|}$ is "non-aesthetic" in that it is not a scalar density of weight one. Instead, it is a strange "object" which transforms under the change of coordinates as a density modulo some additive correction by a total divergence. Nevertheless it works. Hilbert, led by his mathematical intuition, guessed (1.3) immediately as the only geometrically correct possibility (up to the "cosmological" term). Unlike this, the back-breaking attempts by Einstein were rather based on qualitative physical ideas full of mistakes, sometimes quite funny.

The structurally dominant term of (1.3), (1.5) has the form (modulo constant factor):

$$
g^{\mu \nu} g^{\alpha \gamma} g^{\beta \delta}\left(\partial_{\mu} g_{\alpha \beta}\right)\left(\partial_{\nu} g_{\gamma \delta}\right) \sqrt{|g|} .
$$

Obviously, without the next terms, this is a completely non-tensorial expression [13, 14], but it just focuses and visualizes the very essence of nonlinear self-interaction of $g$.

Lagrangian of matter fields, denoted symbolically by $\Psi$, is given by

$$
\mathcal{L}_{\text {matt }}[g, \Psi]=\mathcal{L}_{\text {matt }}(g, \partial g ; \Psi, \partial \Psi) .
$$

The metric $g$ is used here for contracting the tensorial indices of $\Psi$, and its first derivatives $\partial g$ occur in the Levi-Civita affine connection used for tensorially invariant differentiation of $\Psi$. The total Lagrangian

$$
\mathcal{L}_{\text {tot }}[g, \Psi]:=\mathcal{L}_{\mathrm{H}}[g]+\mathcal{L}_{\text {matt }}[g, \Psi]
$$


is invariant under the huge infinite-dimensional group Diff $M$ of all diffeomorphisms of $M$ onto itself,

$$
\mathcal{L}_{\text {tot }}\left[\varphi_{*} g, \varphi_{*} \Psi\right]=\varphi_{*} \mathcal{L}_{\text {tot }}[g, \Psi]
$$

for any $\varphi \in$ Diff $M$. This concerns separately both terms, and obviously the action functional, just as its both terms separately, is invariant in the sense:

$$
I[g, \Psi \mid \Omega]=I\left[\varphi_{*} g, \varphi_{*} \Psi \mid \varphi(\Omega)\right] .
$$

Obviously, the action over the $\Omega$-domain is given by

$$
I[g, \Psi \mid \Omega]=\int_{\Omega} \mathcal{L}[g ; \Psi] \mathrm{d}_{4} x ;
$$

it is a well-defined scalar because $\mathcal{L}$ is a scalar density of weight one. The gravitational Hilbert and matter actions $I_{\mathrm{H}}, I_{\text {matt }}$ are defined separately in the same way. If $\mathcal{L}_{\mathrm{H}}$ is replaced by $G_{\mathrm{H}} \sqrt{|g|}$, then the invariance (1.10) is replaced by the invariance modulo some additive term depending only on the values of fields on the boundary $\partial \Omega$. Obviously, this does not affect the invariance of the Euler-Lagrange field equations.

Let us remind that the field equations have the form:

$$
\begin{aligned}
R_{\mu \nu}-\frac{1}{2} R g_{\mu \nu} & =\varkappa T_{\mu \nu} \\
\frac{\partial \mathcal{L}_{\text {matt }}}{\partial \Psi^{A}}-\frac{D}{D x^{\mu}} \frac{\partial \mathcal{L}}{\partial \Psi^{A}, \mu} & =0
\end{aligned}
$$

where $R_{\mu \nu}=R_{\mu \alpha \nu}^{\alpha}$ is the Ricci tensor, $R=g^{\mu \nu} R_{\mu \nu}$ is the curvature scalar, and $T_{\mu \nu}$ is the metrical (thus, symmetric) energy-momentum tensor of matter,

$$
T_{\mu \nu}=-\frac{2}{\sqrt{|g|}} \frac{\delta I_{\mathrm{matt}}}{\delta g^{\mu \nu}},
$$

where, obviously, one must carefully distinguish between covariant and contravariant components of tensors. In particular, on the right-hand side of (1.13) one performs the variational procedure with respect to the contravariant inverse of $g$.

Without matter, i.e., in empty space-time, field equations reduce to

$$
R_{\mu \nu}=0
$$

and, by analogy to (1.6), the first, leading term has the d'Alembert structure,

$$
g^{\mu \nu} \partial_{\mu} \partial_{\nu} g_{\alpha \beta}+\ldots=0
$$

Of course, this gives a correct insight into the dynamical structure of field equations, but one must remember that the "d'Alembert" term is not to be meant literally, because it has no well-defined tensorial structure.

It is interesting to mention a finite-dimensional counterpart of this framework, the one within the domain of Hamiltonian dynamics 14 18. Namely, let us imagine some physics the area of which is not a general differential manifold, but rather some affine space $M$ with the linear space of translations $V$. Instead of relativistic four-dimensional metric of the normal-hyperbolic signature, we have an Euclidean metric (positive one) in the usual space. Assume this metric to be a dynamical object, and its "kinetic energy" to be an expression of the form:

$$
T[g]=\frac{I}{2} g^{i k} g^{j l} \frac{\mathrm{d} g_{i j}}{\mathrm{~d} t} \frac{\mathrm{d} g_{k l}}{\mathrm{~d} t}+\frac{K}{2} g^{i j} g^{k l} \frac{\mathrm{d} g_{i j}}{\mathrm{~d} t} \frac{\mathrm{d} g_{k l}}{\mathrm{~d} t},
$$

$K, I$ being constants. 
By analogy with Hamiltonian systems on groups, this may be written as:

$$
T[g]=\frac{I}{2} \operatorname{Tr}\left(\Omega_{l}{ }^{2}\right)+\frac{K}{2}\left(\operatorname{Tr} \Omega_{l}\right)^{2}=\frac{I}{2} \operatorname{Tr}\left(\Omega_{r}{ }^{2}\right)+\frac{K}{2}\left(\operatorname{Tr} \Omega_{r}\right)^{2},
$$

where the quantities $\Omega_{l} \in V \otimes V^{*} \simeq \mathrm{L}(V), \Omega_{r} \in V^{*} \otimes V$ are defined as follows:

$$
\Omega_{l}{ }^{a} b:=g^{a c} \frac{\mathrm{d} g_{c b}}{\mathrm{~d} t}, \quad \Omega_{r a}{ }^{b}=\frac{\mathrm{d} g_{a c}}{\mathrm{~d} t} g^{c b} .
$$

Of course, in spite of certain formal similarities, one should be aware of the difference between this model and that of Hamiltonian systems on groups [15, 16, 19 21].

It is easily seen that (1.16), (1.17) is a finite-dimensional toy model of (1.3), (1.5), (1.6). The self-interaction structure of $g$ and its characteristic, non-perturbative nonlinearity is in principle like in the Hilbert principle. As a geodetic model in analytical mechanics, (1.16) is based on the following metric tensor $\mathcal{G}$ on the manifold of all metric tensors in $V$ :

$$
\mathcal{G}=J g^{i k} g^{j l} \mathrm{~d} g_{i j} \otimes \mathrm{d} g_{k l}+K g^{i j} g^{k l} \mathrm{~d} g_{i j} \otimes \mathrm{d} g_{k l} .
$$

This metric tensor is evidently non-Euclidean and the corresponding Riemannian structure on $\operatorname{Sym}\left(V^{*} \otimes V^{*}\right)$ (or rather on its submanifold consisting of the positive metrics $g$ ) has a nonvanishing curvature tensor. By analogy to (1.7), (1.8) one can put $g$ into interaction with "matter", e.g., with the "particle" of mass $m$, moving in $M$, then the total kinetic energy ( $x^{i}$ denoting particle coordinates) is given by

$$
T=T[g]+T_{\text {matt }}[g, x]=\frac{I}{2} g^{i k} g^{j l} \frac{\mathrm{d} g_{i j}}{\mathrm{~d} t} \frac{\mathrm{d} g_{k l}}{\mathrm{~d} t}+\frac{K}{2} g^{i j} g^{k l} \frac{\mathrm{d} g_{i j}}{\mathrm{~d} t} \frac{\mathrm{d} g_{k l}}{\mathrm{~d} t}+\frac{m}{2} g_{i j} \frac{\mathrm{d} x^{i}}{\mathrm{~d} t} \frac{\mathrm{d} x^{j}}{\mathrm{~d} t} .
$$

Obviously, the same may be easily done for the system of particles. Expression (1.20) is based on the following metric tensor $\mathcal{G}$ on $M \times \operatorname{Sym}^{+}\left(V^{*} \otimes V^{*}\right)$ :

$$
\mathcal{G}=m g_{i j} \mathrm{~d} x^{i} \otimes \mathrm{d} x^{j}+J g^{i k} g^{j l} \mathrm{~d} g_{i j} \otimes \mathrm{d} g_{k l}+K g^{i j} g^{k l} \mathrm{~d} g_{i j} \otimes \mathrm{d} g_{k l} .
$$

The analogy is obvious. The "matter" term may be extended by introducing some "potential energy" $V$, e.g., as a function of the "radial" invariant

$$
r^{2}=g_{i j} x^{i} x^{j} .
$$

Kinetic energy (1.20) and its underlying metric (1.21) are invariant under the affine group $\operatorname{GAff}(M)$, which, after the choice of some origin $\mathcal{O} \in M$, may be identified with the semi-direct product $\mathrm{GL}(V) \times V$. The algebraic invariant (1.22) is invariant only under $\mathrm{GL}(V)$, or more precisely, under the centro-affine group $\operatorname{GAff}(M, \mathcal{O}) \subset \operatorname{GAff}(M)$ preserving the origin. When dealing with multi-particle material system in $M$, one can obtain the total affine symmetry, replacing the quantity (1.22) by the system of translationally-invariant functions $r_{A B}$ on the configuration space $Q=M \times \operatorname{Sym}^{+}\left(V^{*} \otimes V^{*}\right)$; these "radial-like" quantities are defined as

$$
r_{A B}=\sqrt{g_{i j}\left(x^{i}{ }_{A}-x_{B}^{i}\right)\left(x^{j}{ }_{A}-x_{B}^{j}\right)},
$$

where $x^{i}{ }_{A}$ are affine coordinates of the $A$-th particle. It is seen that the symmetry $\operatorname{GAff}(M)$ is analogous to the general covariance Diff $M$ of General Relativity and those are just those huge symmetry groups which are "responsible" for the strong, non-perturbative nonlinearities of the models. The group GAff $(M)$ acts on metrical degrees of freedom through its quotient linear group $\mathrm{GL}(V)$, in the sense

$$
A \in \mathrm{GL}(V): \quad\left[g_{i j}\right] \mapsto\left[\left(A_{*} g\right)_{i j}\right]=\left[g_{k l} A_{i}^{-1}{ }_{i}^{k} A_{j}^{-1}{ }_{j}^{l} .\right.
$$


Let us mention that there are also some other generalized potentials, i.e., the ones depending on generalized velocities, compatible with those invariance demands. But there is no place here for analyzing this problem more in detail.

Let us now concentrate on something else. In the toy models discussed above we were dealing with models in analytical mechanics which had two kinds of degrees of freedom: translational ones in the physical affine space and internal ones, represented by the metric tensor as a kind of collective variable. This picture remains with certain instructive analogy to the metrical (generallyrelativistic) model of gravitation. Analytically, the internal modes were described by matrices. But we also mentioned another class of mechanical models, namely, the one where internal/collective degrees of freedom were represented by elements of some Lie groups, to be more precise, by elements of linear Lie groups, or by other manifolds of linear mappings. Analytically they are also represented by matrices. However, and this is geometrically important, they are other objects, namely, mixed tensors, whereas scalar products are twice covariant tensors. Objects with spaces of mixed tensors as configuration spaces were investigated, e.g., by us and others in mechanics of so-called affinelyrigid bodies. And they may be considered as mechanical toy models of certain alternative models of gravitation, namely, tetrad models in their various versions. And this has again very much, just even more, to do with the link between symmetry and nonlinearity, and with some more or less diffused interpenetration between field theory and condensed matter physics, in particular, relativistic mechanics of structured continua.

Let us again go back to gravitation ideas and relativistic structured media, this time within the framework of more or less modified tetrad models [7, 8, 22]. Historically, the tetrad models, originating from Weyl and Einstein, were thought to be some bridge between specially- and generally-relativistic theories. Later on, it turned out that they provide much richer class of models than the material Hilbert/Einstein model. And the most deciding point was the Dirac theory of generally relativistic spinors, impossible to be formulated within the metrical framework. The main reason is that the universal covering group of $\mathrm{GL}(4, \mathbb{R})$ unlike $\mathrm{GL}(4, \mathbb{R})$ itself, is not linear, i.e., cannot be faithfully realized by finite matrices. The same is true for any dimension $n \geqslant 3$.

Instead of the metric field $g$, one uses the field of linear frames $e=\left(\ldots, e_{A}, \ldots\right)$ as the system of gravitational potential. Equivalently, one can use the dual field of co-frames $e^{-1}=\left(\ldots, e^{A}, \ldots\right)$, where

$$
\left\langle e^{A}, e_{B}\right\rangle=e_{\mu}^{A} e_{B}^{\mu}=\delta_{B}^{A} ;
$$

they uniquely determine each other.

The next object is the corresponding teleparallelism connection $\Gamma[e$, tel] [8, 13, 14, 22], defined uniquely by the condition that $e$ is parallel with respect to the corresponding covariant differentiation $\nabla[e$, tel $]$ :

$$
\nabla e_{A}=0, \quad a=1, \ldots n .
$$

When no ambiguity occurs, we use the abbreviation $\Gamma[$ tel], $\nabla[$ tel], or just $\Gamma, \nabla$. One proves immediately that in local coordinates

$$
\Gamma[e, \text { tel }]^{\mu}{ }_{\nu \lambda}=e^{\mu}{ }_{A} e^{A}{ }_{\nu, \lambda}=-e^{\mu}{ }_{A, \lambda} e^{A}{ }_{\nu} .
$$

Obviously, the curvature tensor of $\Gamma[t e l]$ vanishes, but in general its torsion

$$
S[e, \text { tel }]_{\nu \lambda}^{\mu}=\frac{1}{2} e_{A}^{\mu}\left(e_{\nu, \lambda}^{A}-e_{\lambda, \nu}^{A}\right)
$$

is a non-vanishing tensor; incidentally, it is well-known in the theory of dislocations. Here it plays the role of tensorially invariant derivative of the field $e$.

Let $\eta$ denote some symmetric non-degenerate metric tensor in the target space of the field $e$. Analytically it is given by a constant and nonsingular symmetric matrix $\left[\eta_{A B}\right]$. For physical reasons its signature is normal-hyperbolic; in the physical dimension $n=4$, we usually put it as

$$
\left[\eta_{A B}\right]=\operatorname{Diag}(1,-1,-1,-1)
$$


The contravariant inverse is denoted by $\left[\eta^{A B}\right]$ :

$$
\eta_{A C} \eta^{C B}=\delta_{A}^{B} .
$$

The Weyl-Dirac-Einstein metric tensor is given by

$$
h[e]=\eta_{A B} e^{A} \otimes e^{B}, \quad h[e]_{\mu \nu}=\eta_{A B} e^{A}{ }_{\mu} \otimes e^{B}{ }_{\nu} .
$$

The linear group $\mathrm{GL}(n, \mathbb{R})(\mathrm{GL}(4, \mathbb{R})$ physically), as a structural group of the principal bundle of linear frames $F M$ or its dual $F^{*} M$, physically is a group of internal transformations of the field $e$ (or dually, $e^{-1}$ ). It acts according to the rule:

$$
\begin{aligned}
e=\left(\ldots, e_{A}, \ldots\right) & \mapsto e L=\left(\ldots, e_{B} L^{B}{ }_{A}, \ldots\right), \\
e^{-1}=\left(\ldots, e^{A}, \ldots\right) & \mapsto(e L)^{-1}=\left(\ldots, L^{-1}{ }_{B} e^{B}, \ldots\right),
\end{aligned}
$$

for arbitrary $L \in \mathrm{GL}(n, \mathbb{R})$. This is a global action, and in geometry one considers also the local action of the fields $L: M \rightarrow \mathrm{GL}(n, \mathbb{R})$, according to the rule:

$$
e(x) \mapsto e(x) L(x), \quad e(x)^{-1} \mapsto(e(x) L(x))^{-1} .
$$

One can also restrict the values of $L$ to the Lorentz subgroup $\mathrm{O}(n, \eta) \subset \mathrm{GL}(n, \mathbb{R})$ (physically $\mathrm{O}(1,3) \subset \mathrm{GL}(4, \mathbb{R}))$, when

$$
\eta_{C D} L^{C}{ }_{A} L^{D}{ }_{B}=\eta_{A B} \text {. }
$$

Obviously, $h[e]$ is invariant under the local Lorentz action (1.33), (1.34). Unlike this, $S[e]$ is invariant only under the global ( $x$-independent) action (1.32) of the total $\mathrm{GL}(n, \mathbb{R})$ and its Lorentz subgroup. But both the prescription $e \mapsto h[e]$ and $e \mapsto S[e]$ are generally covariant,

$$
h\left[\varphi_{*} e\right]=\varphi_{*} h[e], \quad S\left[\varphi_{*} e\right]=\varphi_{*} S[e] .
$$

Using the tensors $h[e], S[e]$ as algebraic brick-stones, one can construct some byproduct quantities. First of all, let us quote some tensors built of the tensor $S[e]$ alone:

$$
\begin{aligned}
\gamma_{\mu \nu} & =4 S^{\alpha}{ }_{\mu \beta} S^{\beta}{ }_{\nu \alpha}=\gamma_{\nu \mu}, \quad \gamma_{\mu}=S^{\alpha}{ }_{\mu \alpha}, \\
\Gamma_{\mu \nu} & =4 S^{\alpha}{ }_{\beta \alpha} S^{\beta}{ }_{\mu \nu}=-\Gamma_{\nu \mu}=2 \gamma_{\alpha} S^{\alpha}{ }_{\mu \nu} .
\end{aligned}
$$

These are the only tensors built algebraically of $S$ alone in a quadratic or linear $\left(\gamma_{\mu}\right)$ way.

Another important quantities are scalars built in a quadratic way of $S$, with coefficients built algebraically of $h[e]$, so-called Weitzenböck invariants [7, [8, 22]:

$$
\begin{aligned}
& J_{1}=h_{\alpha \mu} h^{\beta \nu} h^{\gamma \varkappa} S_{\beta \gamma}^{\alpha} S^{\mu}{ }_{\nu \varkappa}, \\
& J_{2}=\frac{1}{4} h^{\mu \nu} \gamma_{\mu \nu}=h^{\mu \nu} S^{\alpha}{ }_{\mu \beta} S^{\beta}{ }_{\alpha \nu}, \\
& J_{3}=\frac{1}{4} h^{\mu \nu} \gamma_{\mu} \gamma_{\nu}=h^{\mu \nu} S^{\alpha}{ }_{\mu \beta} S^{\beta}{ }_{\nu \alpha} .
\end{aligned}
$$

There are also plenty of other concomitants of $S[e], h[e]$, but the above ones are distinguished by their property of be quadratic in derivatives of $e$ (with the exception of $\gamma_{\mu}$, which is linear in derivatives). No doubt, such quantities are geometrically distinguished when constructing Lagrangians. Let us notice the particular role of $\gamma_{\mu \nu}$. Being symmetric, it is an alternative candidate for the metric tensor of $M$; i.e., alternative with respect to the Einstein-Weyl-Dirac metric $h[e]$. It is still more important that if $\left(\ldots, e_{A}, \ldots\right)$ form a semi-simple Lie algebra with respect to the Lie bracket,

$$
\left[e_{A}, e_{B}\right]=C^{K}{ }_{A B} e_{K},
$$


$C_{A B}^{K}$ being (structure) constants such that

$$
\operatorname{det}\left[C^{K}{ }_{A L} C^{L}{ }_{B K}\right] \neq 0,
$$

then locally $M$ may be identified with a semi-simple Lie group, and $\gamma[e]_{\mu \nu}$ becomes its nondegenerate Killing tensor [13, 14]. This is interesting, the more so that the signature of $\gamma_{\mu \nu}$ is not introduced "by hand"; instead it is a consequence of something more fundamental.

It is important that all the above quantities are built of the field $e$ in a generally-covariant way, so they satisfy

$$
F\left[\varphi_{*} e\right]=\varphi_{*} F[e], \quad \varphi \in \operatorname{Diff} M
$$

Their invariance status under internal transformations GL $(n, \mathbb{R})$, operating on the capital indices (target space transformations) is a more complicated matter. Certainly the torsion tensor $S$, the Killing and similar objects like (1.36), built algebraically of $S$ alone, are invariant under the global action of GL $(n, \mathbb{R}),(1.32)$. The same concerns, of course, all Diff $M$-invariant scalars built of $S$ alone. Incidentally, one can show that all such scalars are homogeneous functions of degree zero built of $S$. The dependence of the Dirac-Weyl-Einstein metric $h[e]$ of $e$ is invariant under the local action of $\mathrm{O}(1,3) \subset \mathrm{GL}(4, \mathbb{R})(\mathrm{O}(n, \eta) \subset \mathrm{GL}(n, \mathbb{R}))$, i.e., under (1.33) with values of $L$ restricted to the Lorentz subgroup. Certainly $e \mapsto h[e]$ is not invariant under GL $(4, \mathbb{R})(\mathrm{GL}(n, \mathbb{R}))$ even in the global sense. Moreover, it is not invariant under any subgroup of $\mathrm{GL}(4, \mathbb{R})$ larger than the Lorentz group. The same concerns the Weitzenböck invariants (1.37). There is, however, some delicate point with very important and far-reaching consequences. Namely, substituting to the Hilbert Lagrangian (1.3) the metric $h[e]$ instead of $g$, one obtains the following expression for $\mathcal{L}_{\mathrm{H}}$ as a function of $(e, \partial e)$ :

$$
\mathcal{L}_{\mathrm{H}}[e]=-\frac{1}{2 \varkappa}\left(J_{1}+2 J_{2}-4 J_{3}\right) \sqrt{|h[e]|}-\frac{2}{\varkappa} \partial_{\mu}\left(S^{\alpha}{ }_{\alpha \beta} h^{\beta \mu} \sqrt{|h|}\right) .
$$

The fourth term is a well-defined scalar density of weight one, because it is a usual partial-derivative divergence of the contravariant vector density of weight one. The symbol $\partial_{\mu}$ in this term may be replaced by the covariant derivative $\nabla_{\mu}$ with respect to the Levi-Civita affine connection built of $h[e]$. This fourth term absorbs all second derivatives $\partial^{2} e$ of the basic field $e$. Being a total divergence, it may be simply neglected. Then we obtain the effective Lagrangian explicitly free of second derivatives,

$$
\mathcal{L}[e]=-\frac{1}{2 \varkappa}\left(J_{1}+2 J_{2}-4 J_{3}\right) \sqrt{|h[e]|} .
$$

It is very important that unlike $G_{\mathrm{H}} \sqrt{|g|}$ in (1.5), the expression (1.42) is a well-defined scalar density of weight one. It is generally-covariant,

$$
\mathcal{L}\left[\varphi_{*} e\right]=\varphi_{*} \mathcal{L}[e], \quad \varphi \in \operatorname{Diff} M .
$$

As concerns the total action of the internal Lorentz group, i.e., (1.33) with $L(x)$ satisfying (1.34) for any $x \in M$, obviously (1.42) is variationally invariant, i.e., invariant modulo some divergence term.

Once derived, (1.42) may be generalized to a wide class of Lagrangians. First of all, the ratio

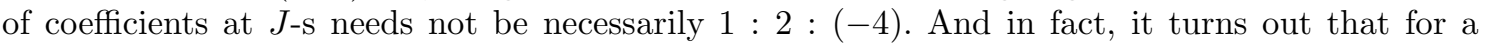
wide range of coefficients $c_{1}, c_{2}, c_{3}$ at $J_{1}, J_{2}, J_{3}$ such modified Lagrangians are compatible with experimental data. The more serious modifications consist in admitting Lagrangians depending in a general, including nonlinear, way on the Weitzenböck invariants,

$$
\mathcal{L}[e]=f\left(J_{1}, J_{2}, J_{3}\right) \sqrt{|h[e]|},
$$

$f$ being some real function of three variables. Nonlinearity of such models may be incomparatively stronger than that of tetrad Hilbert model (1.42) or its modified-coefficients version:

$$
\mathcal{L}[e]=\left(c_{1} J_{1}+c_{2} J_{2}+c_{3} J_{3}\right) \sqrt{|h|} .
$$


Historically, there were some attempts to avoid certain difficulties of General Relativity, by admitting the form (1.44) 22].

Obviously, models (1.44) other than (1.42) are only globally, no longer locally, invariant under the internal Lorentz group. But having once admitted such models, one is immediately faced with the very natural temptation: why not try to construct generally-covariant Lagrangians $\mathcal{L}[e]$ invariant under the total linear group $\operatorname{GL}(4, \mathbb{R})$ (perhaps $\operatorname{GL}(n, \mathbb{R})$ in $n$-dimensional space-times)? This is a very natural idea, because a priori $\operatorname{GL}(4, \mathbb{R})(\operatorname{GL}(n, \mathbb{R}))$, the structure group of the principal bundle of frames, is the most natural internal group, while any restriction of $\operatorname{GL}(n, \mathbb{R})$ to a subgroup seems to be non-motivated by any "first principles". Any Lagrangian invariant under Diff $M$ and $\operatorname{GL}(n, \mathbb{R})$ must be built algebraically of $S$ alone. One can show that it is always a homogeneous function of degree $n=\operatorname{dim} M$ of the tensor $S$. And now, something very interesting results. It turns out that Lagrangians of this type have automatically a generalized Born-Infeld structure $7-10]$. The simplest of them have the form:

$$
\mathcal{L}[e]=\sqrt{\left|\operatorname{det}\left[L_{\mu \nu}\right]\right|},
$$

where $L_{\mu \nu}$, referred too as the Lagrangian tensor, is a linear combination of tensors $\gamma_{\mu \nu}, \gamma_{\mu} \gamma_{\nu}$, and $\Gamma_{\mu \nu}$, cf. (1.36):

$$
L_{\mu \nu}=A \gamma_{\mu \nu}+B \gamma_{\mu} \gamma_{\nu}+C \Gamma_{\mu \nu}
$$

where $A, B, C$ are real constants. One can also admit a purely imaginary $C$. Then the tensor $L_{\mu \nu}$ is hermitian, $L_{\mu \nu}=\overline{L_{\nu \mu}}$, and its determinant is real. There also exist more complicated models, where $A, B, C$ are scalar functions of $S$. One can also multiply the total square root by some scalars built of $S$ alone. One can show that generally-covariant scalars built algebraically of $S$ alone are always homogeneous functions of degree zero. Typical structure of such scalars is as follows: If $\gamma_{\mu \nu}$ happens to be non-degenerate, then it may be used to the raising of indices. Then one can construct of (1.36) various mixed tensors, their traces, etc; in a natural way scalars may be obtained on the basis of trace-taking. Introducing such scalars into (1.46), (1.47) would drastically complicate the model, but probably without a serious chance of obtaining something essentially new.

There are some very interesting peculiar features of the model (1.46), (1.47). Namely, it is clear that Lagrangian $\mathcal{L}$, i.e., integrand of the action functional, is a scalar $W$-density of weight one. This is necessary if the action $I$ (1.11) is to be a scalar quantity, and obviously, physically it should be so. There is one, almost canonical prescription for obtaining such densities. Namely, scalar densities of weight two usually appear as determinants of matrices of twice covariant tensors. Then the scalar $W$-densities are obtained as square roots of the absolute values of those determinants. Another approach is to interpret Lagrangian as a differential $n$-form. Those are in principle equivalent formulations. However, there are some subtle points concerning the orientation of $M$.

In any case, it is quite natural to say that the primary notation of variational theory is Lagrange tensor $L_{\mu \nu}$ depending algebraically on a given field $\Psi$, its derivatives $\partial \Psi$ and eventually on the space-time point $x$,

$$
L_{\mu \nu}(x, \Psi(x), \partial \Psi(x))
$$

What is usually referred to as Lagrangian $\mathcal{L}$, is a by-product, scalar density of weight one, given by (1.46). In commonly used theories, one uses some metric tensor $g_{\mu \nu}$ and the scalar representation $L$ of $\mathcal{L}$ given by the factorization:

$$
\mathcal{L}=L \sqrt{|g|}
$$

This is the language used in General Relativity.

The "Born-Infeld" property of (1.46) is that it is the square-root of the determinant of "something", and the "something", i.e., Lagrange tensor, is a low-order, in this case second-order polynomial of field derivatives.

In traditional Born-Infeld electrodynamics, Lagrangian has the form [9, 10]:

$$
\mathcal{L}=b^{2} \sqrt{\left|\operatorname{det}\left[g_{\mu \nu}\right]\right|}-\sqrt{\left|\operatorname{det}\left[b g_{\mu \nu}+F_{\mu \nu}\right]\right|},
$$


where, obviously, the first term is non-dynamical. In Special Relativity it is simply constant; in General Relativity it is $x$-dependent, but still independent of the electromagnetic field. Dynamical quantities of the theory are $A_{\mu}$, i.e., components of the four-potential covector; $F_{\mu \nu}$ is the electromagnetic tensor,

$$
F_{\mu \nu}=\partial_{\mu} A_{\nu}-\partial_{\nu} A_{\mu}
$$

The only, rather artificial, role of the first term in (1.50) is to make the Lagrangian and energy vanishing when $F$ does vanish. The true dynamics is encoded purely in the second term. The Lagrange tensor is given by

$$
L_{\mu \nu}=b g_{\mu \nu}+F_{\mu \nu},
$$

so, it is a first-order polynomial of derivatives of dynamical fields. It is just the peculiarity of the electromagnetic field that linear (or rather affine, linear-inhomogeneous) Lagrange tensors do exist, although some alternative models with quadratic dependence on derivatives may be also constructed. In general, the quadratic dependence on derivatives is the simplest possibility. The model (1.50) was motivated by certain difficulties of classical electrodynamics. Due to the squareroot structure, it predicts the saturation of electromagnetic field, by analogy to the maximal velocity, i.e., velocity of light in relativistic point mechanics. Because of this, the energy of point charges, i.e., electromagnetic mass, was finite. For many physical reasons, the model (1.50), (1.52) is a canonical nonlinearity compatible with electrodynamics [9, 11]. Incidentally, in spite of certain current views, classical electrodynamics is still full of mysteries. Born-Infeld nonlinearity has to do with many of them [1, 5, 6]. Let us notice, however, that in (1.46), (1.47) the "Born-Infeld" structure follows from something very fundamental, namely, from the invariance assumptions: The model was to be generally-covariant, i.e., invariant under Diff $M$, and invariant under GL $(n, \mathbb{R})$ (physically $\mathrm{GL}(4, \mathbb{R}))$ as internal symmetry group. The "huge" symmetry Diff $M \times \operatorname{GL}(n, \mathbb{R})$ just implies the "Born-Infeld" nonlinearity, i.e., self-interaction, as the simplest possible model. Lagrange tensor (1.47) is quadratic in derivatives of field variables. In a sense, this is another "pole" of physical "simplicity", alternative to linearity. In linear (and quasilinear) models, Lagrangians are quadratic in derivatives. In "Born-Infeld" models it is no longer Lagrangian, but Lagrange tensor that is quadratic (sometimes linear) in derivatives. In these models one deals with the essential, nonperturbative nonlinearity, i.e., essential, strong self-interaction. This self-interaction is deeply based on geometry. And due to this geometric background and the underlying symmetry group, the resulting nonlinearity is not artificially complicated. As concerns simplicity, it is as close to linear systems as possible. This kinship is based on the alternative:

\section{Quadratic Lagrange Tensor - Quadratic Lagrangian.}

In a sense, in tetrad models, the opposition between field theory and continuum theory (condensed matter) diffuses. From some point of view, the tetrad field is a gravitational potential, but at the same time it may be physically interpreted as the relativistic micromorphic continuum. Roughly speaking, integral curves of the time-like "legs" of tetrads are world-lines of continuum particles. The remaining "legs" represent internal degrees of freedom of this continuum, attached frames. So, it is really something like micropolar (Cosserat) or micromorphic (Eringen) continuum medium $[2,3,[23,24]$.

Now, let us close the circle of analogies in our study of essential nonlinearities and geometric self-interactions. From General Relativity we passed to its finite-dimensional models based on (1.16), (1.17), (1.19)-(1.21), etc., where the "spatial" metric $g$ was a kind of the internal/collective variable. Then, the tetrad models of gravitation were briefly discussed, with the special stress on the Born-Infeld type of self-interaction. Let us go back to finite-dimensional analytical mechanics. There is an analogy between transition from General Relativity to tetrad models and the transition from (1.16), (1.17), (1.19)-(1.21) to the so-called affinely-rigid bodies, i.e., bodies rigid in the sense of affine geometry 25 51]. The configuration space of internal/collective modes will be given then by $\mathrm{F}(V)$, the manifold of linear frames in $V$. So, the configuration space $M \times \mathrm{Sym}^{+}\left(V^{*} \otimes V^{*}\right)$ will be replaced by $M \times \mathrm{F}(V)$. Generalized coordinates are $\left(x^{i}, e_{A}^{i}\right)$, where $e^{i}{ }_{A}$ are components of the frame vectors $e_{A}$ with respect to spatial coordinates $x^{i}$. By analogy with (1.31) one can use the 
metric

$$
h[e]=\delta_{A B} e^{A} \otimes e^{B}, \quad h[e]_{i j}=\delta_{A B} e_{i}^{A} e_{j}^{B},
$$

where, obviously, $\left(\ldots, e^{A}, \ldots\right)$ is the co-frame dual to $\left(\ldots, e_{A}, \ldots\right)$. If some metric tensor $g \in$ $V^{*} \otimes V^{*}$ is fixed, then the usual reasoning leads to the following kinetic energy form:

$$
T=\frac{m}{2} g_{i j} \frac{\mathrm{d} x^{i}}{\mathrm{~d} t} \frac{\mathrm{d} x^{j}}{\mathrm{~d} t}+\frac{1}{2} g_{i j} \frac{\mathrm{d} e^{i} A}{\mathrm{~d} t} \frac{\mathrm{d} e^{j} B}{\mathrm{~d} t} J^{A B},
$$

where $J^{A B}$ is the co-moving, thus, constant, tensor of inertia, i.e., quadrupole momentum of the mass distribution in representation of co-moving axes given by the moving frame $e=\left(\ldots, e_{A}, \ldots\right)$ [7, $8,28,50,52,56$. If the mass distribution within the body is isotropic with respect to the co-moving frame, then

$$
J^{A B}=I \delta^{A B} ;
$$

this is an affine analogue of the spherical rigid body.

Kinetic energy (1.54) is invariant under the Euclidean group $\mathrm{E}(M, g)$, in particular, under spatial translations and under orthogonal group $\mathrm{O}(V, g) \subset \mathrm{GL}(V)$; more precisely, under its centroaffine versions $\mathrm{E}(M, g ; \mathcal{O})$, where $\mathcal{O} \in M$ is a fixed origin in $M$. It is also invariant under the internal (material) $J$-orthogonal group $\mathrm{O}(n, J) \subset \mathrm{GL}(n, \mathbb{R})$. The latter group consists of matrices $L$ such that

$$
L^{A}{ }_{C} L^{B}{ }_{D} J^{C D}=J^{A B} .
$$

If $J$ is isotropic, then (1.55) holds and $\mathrm{O}(n, J)$ becomes just the usual orthogonal group $\mathrm{O}(n, \mathbb{R})$.

In formulas (1.16), (1.17) we just objected against the fixed $g$; it was to be dynamical. If we follow the ideas of tetrad theory of gravitation, then it seems natural to do something else, namely, to substitute in (1.54) $h[e]$ instead of $g$,

$$
T=\frac{m}{2} h[e]_{i j} \frac{\mathrm{d} x^{i}}{\mathrm{~d} t} \frac{\mathrm{d} x^{j}}{\mathrm{~d} t}+\frac{1}{2} h[e]_{i j} \frac{\mathrm{d} e^{i} A}{\mathrm{~d} t} \frac{\mathrm{d} e^{j} B}{\mathrm{~d} t} J^{A B} .
$$

This may be written as follows:

$$
T=\frac{m}{2} \delta_{A B} \widehat{v}^{A} \widehat{v}^{B}+\frac{1}{2} \delta_{A B} \widehat{\Omega}^{A}{ }_{C} \widehat{\Omega}^{B}{ }_{D} J^{C D},
$$

where $\widehat{v}^{A}$ and $\widehat{\Omega}^{K}{ }_{L}$ are $e$-co-moving components of translational velocity and the so-called affine velocity, respectively:

$$
\widehat{v}^{A}=e^{A}{ }_{i} \frac{\mathrm{d} x^{i}}{\mathrm{~d} t}=e^{A}{ }_{i} v^{i}, \quad \widehat{\Omega}^{K}{ }_{L}=e^{K}{ }_{i} \frac{\mathrm{d} e^{i} L}{\mathrm{~d} t} .
$$

The corresponding spatial affine velocity $\Omega^{i}{ }_{j}$ is defined and related to $\widehat{\Omega}$ as follows:

$$
\Omega^{i}{ }_{j}=\frac{\mathrm{d} e^{i} K}{\mathrm{~d} t} e^{K}{ }_{j}=e^{i}{ }_{K} \widehat{\Omega}^{K}{ }_{L} e^{L}{ }_{j} .
$$

$\Omega, \widehat{\Omega}$ are Lie-algebraic objects, affine counterparts of angular velocity. Let us notice that $h[e]$ is identical with the Cauchy deformation tensor of elasticity theory, or rather, its special case corresponding to homogeneous (affine) deformations.

It is very interesting that (1.57), (1.58) is invariant under GAff $(M)$, the total affine group in $M$. This is a finite-dimensional counterpart of Diff $M$, the group of general covariance in (1.3), (1.42), (1.44), (1.45). And if $J^{A B}=I \delta^{A B}$ (isotropy of the inertial tensor), then (1.58) becomes

$$
T=T_{\mathrm{tr}}+T_{\mathrm{int}}=\frac{m}{2} \delta_{A B} \widehat{v}^{A} \widehat{v}^{B}+\frac{I}{2} \delta_{A B} \widehat{\Omega}^{A}{ }_{C} \widehat{\Omega}^{B}{ }_{D} \delta^{C D}
$$

and in addition to the spatial GAff $(M)$-invariance, we have the "internal" invariance under $\mathrm{O}(n, \mathbb{R})$, the orthogonal group in $n$ dimensions. This is an analogue of the global internal Lorentz invariance in (1.44), (1.45). Physically, the $\mathrm{F}(V)$-degrees of freedom are collective/internal variables, which 
are in a sense more "subtle" than $\operatorname{Sym}^{+}\left(V^{*} \otimes V^{*}\right)$. They introduce affine invariance and the corresponding essential nonlinearity. The question arises as to the finite-dimensional analogues of something like (1.46), (1.47). In other words: how can we extend the internal orthogonal symmetry $\mathrm{O}(n, \mathbb{R})$ of $(1.61)$ to the full linear group $\mathrm{GL}(n, \mathbb{R})$, by analogy with extending the Lorentz internal symmetry of (1.44), (1.45) to the full linear one like in (1.46), (1.47)? To be honest, this is impossible for the total kinetic energy of affine body. It may be affinely invariant either in $M$ or internally in $\mathbb{R}^{n}$, but not simultaneously in both spaces. But the internal part may be affinely invariant both in $M$ and in $\mathbb{R}^{n}$; roughly speaking, simultaneously left and right affinely invariant.

And such models were already briefly mentioned after the formula (1.16). Namely, they are built of the second and first Casimir invariants,

$$
T[e]=\frac{I}{2} \operatorname{Tr}\left(\Omega^{2}\right)+\frac{K}{2}(\operatorname{Tr} \Omega)^{2}=\frac{I}{2} \operatorname{Tr}\left(\widehat{\Omega^{2}}\right)+\frac{K}{2}(\operatorname{Tr} \widehat{\Omega})^{2} .
$$

The corresponding metric tensor on $\mathrm{F}(V)$ is given by

$$
\mathcal{G}=I \omega^{i}{ }_{j} \otimes \omega^{j}{ }_{i}+K \omega_{i}^{i} \otimes \omega^{j}{ }_{j}=I \widehat{\omega}^{A}{ }_{B} \otimes \widehat{\omega}^{B}{ }_{A}+K \widehat{\omega}_{A}^{A} \otimes \widehat{\omega}^{B}{ }_{B},
$$

where $I, K$ are constants and $\omega^{i}{ }_{j}, \widehat{\omega}^{A}{ }_{B}$ are differential forms on $\mathrm{F}(V)$ given respectively by the following formulas:

$$
\omega^{i}{ }_{j}=e^{A}{ }_{j} \mathrm{~d} e^{i}{ }_{A}, \quad \widehat{\omega}^{A}{ }_{B}=e^{A}{ }_{i} \mathrm{~d} e^{i}{ }_{B},
$$

thus, they are interrelated by

$$
\omega^{i}{ }_{j}=e^{i}{ }_{A} e^{B}{ }_{j} \widehat{\omega}^{A}{ }_{B} .
$$

Obviously, the main term is that controlled by $I$; the $K$-term is merely a correction, just by a complete analogy with (1.17), (1.18).

The metric tensor $\mathcal{G}(1.63)$ on the manifold of frames $\mathrm{F}(V)$ is essentially Riemannian, its curvature tensor is non-vanishing. At the same time, this metric has a large isometry group $\operatorname{GL}(V) \times$ $\mathrm{GL}(n, \mathbb{R})$, or rather its quotient with respect to the non-effectiveness kernel

$$
\left\{\left(\lambda I d_{V}, \lambda^{-1} I_{n}\right): \lambda \in \mathbb{R}, \lambda \neq 0\right\} .
$$

Obviously, in the last formula, $I d_{V}$ denotes the identity mapping in $V$, and $I_{n}$ is the $n \times n$ identity matrix.

The large isometry group is, as usual, correlated with the essential nonlinearity, i.e., essential self-interaction of the geodetic problem based on (1.62), (1.63). This is a finite-dimensional pattern for the field-theoretic models (1.46), (1.47). Affine symmetry and the absence of any metrical background in $M$ or $V$ are their common structural features.

It turns out that dynamical models based on (1.46), (1.47) and (1.62) possess certain interesting solutions [7, 8, 28 50, 52 56]. The ones for (1.46), (1.47) admit certain cosmological interpretation. They are also interesting from the point of view of relativistic structured continuum. Geodetic models on $\mathrm{F}(V)$ or $\mathrm{GL}(V)$ based on (1.62), (1.63) are applicable in nonlinear elasticity. This is particularly suggestive when $\mathrm{GL}(V)$ is constrained to $\mathrm{SL}(V)$ or, equivalently, when in $\mathrm{F}(V)$ we impose holonomic constraints according to which the volume of frames is preserved. In anholonomic language such constraints may be described by any of the two equivalent conditions:

$$
\operatorname{Tr} \Omega=0, \quad \operatorname{Tr} \widehat{\Omega}=0 .
$$

It turns out that although the group $\mathrm{SL}(V)$ is non-compact, and so is its any orbit in $\mathrm{F}(V)$, the geodetic models based on (1.62) as a Lagrangian predict some open family of bounded solutions describing nonlinear elastic vibrations, even without any use of potential energy. Above some threshold there is an open set of non-bounded "escaping" solutions ("dissociation threshold"). Such a model is interpretable from the point of view of integrable one-dimensional latices. The "lattice points" on $\mathbb{R}$ appear as deformation invariants. Without incompressibility constraints (1.67) (isochoric motion) the "volume" of $e$ is either constant or behaves in a singular way, collapsing to the point or infinitely expanding. However, this effect may be stabilized by introducing some auxiliary potential depending only on $\operatorname{det}\left[e^{i}{ }_{A}\right]$ and preventing both the collapse and decay.

The usefulness of the geodetic (no-potential) model of elastic vibrations consists in that its analysis may be to some extent reduced to calculating matrix exponents [7, 8, 28, 50, 52 56]. 


\section{General covariance versus Born-Infeld nonlinearity}

Our idea here is that there exists some link between essential, non-perturbative nonlinearity and invariance under "large" symmetry groups. More precisely, nonlinearities following from invariance demands turn out to be physically most interesting. The general covariance, i.e., invariance under Diff $M$, so fundamental for General Relativity, is the best known example. Nonlinearity of Euler equations for ideal fluids is intimately connected with the invariance under the group of all volume-preserving diffeomorphisms of $\mathbb{R}^{3}[16,57]$. The characteristic Born-Infeld-type nonlinearity of our "tetrad" models (1.46), (1.47) is implied by the joint demand of general covariance and the invariance under $\mathrm{GL}(4, \mathbb{R})(\mathrm{GL}(n, \mathbb{R}))$, i.e., internal invariance. Both models have some finitedimensional counterparts, namely, (1.16)-(1.21) and (1.57), (1.61), (1.62). Analytical mechanics offers here some toy models of general covariance and internal symmetry. Incidentally, those toy models may be quite practically useful as description of some internal or collective degrees of freedom.

Two main ideas did appear: general covariance and Born-Infeld structure. Apparently, they seem to be different things, in spite of some generally-relativistic motivation for the electrodynamical Born-Infeld ideas. Nevertheless, the Born-Infeld structure of (1.46), (1.47) was just a direct consequence of the demand of invariance under Diff $M \times \mathrm{GL}(n, \mathbb{R})$ (physically $n=4$ ). Is this accidental?

A more general question arises as to the very relationship between general covariance and the Born-Infeld type of nonlinearity, namely, the characteristic square-root structure and the secondorder polynomial dependence of the Lagrange tensor $L_{\mu \nu}$ on field derivatives. In our model (1.46), (1.47) it was a quite canonical kinship. How is it in general?

Let us begin with the very idea of general covariance. Not every kind of physical field does admit a generally-covariant variational principle. The twice covariant tensor, e.g., metric tensor, does it. And every system of fields containing metric does so as well. This is the very idea of General Relativity. And there was a wrong view that it is the only possibility. Obviously, the tetrad ( $n$-leg) field is also good from this point of view. But what are other possibilities? The peculiarity of the metric field is that it is a twice covariant tensor field, i.e., field of scalar products. What about mixed second-order tensors, i.e., fields of linear mappings? Let $X$, analytically $X^{\mu}{ }_{\nu}$ be such a field. It turns out that $X$ does admit a generally-covariant variational principle, but the simplest thing one can invent is rather complicated and the only possibility is just of the "Born-Infeld" type. Namely, it is a well-known fact that with every pair of mixed (once contravariant and once covariant) tensor fields $X, Y$ one can associate the so-called Nijenhuis torsion $S(X, Y)$, which is once contravariant, twice covariant and antisymmetric in covariant indices. Perhaps it would be rather obscuring to quote the explicit formula, which belongs to the realm of advanced differential geometry [13]; in any case the point is that $S(X, Y)$, analytically $S(X, Y)^{\mu}{ }_{\nu \lambda}$, is algebraically built of the components of $X, Y$ and their first-order derivatives. For any vector field $X$ we can invariantly define the tensor field $S(X):=S(X, X)$ and its byproducts, like, e.g., the Lagrange tensor

$$
L[X]_{\mu \nu}=A S^{\lambda}{ }_{\mu \varkappa} S^{\varkappa}{ }_{\nu \lambda}+B S^{\lambda}{ }_{\mu \lambda} S^{\varkappa}{ }_{\nu \varkappa}+C S^{\lambda}{ }_{\varkappa \lambda} S^{\varkappa}{ }_{\mu \nu},
$$

where $A, B, C$ are real constants. Nothing more natural ("more clever") may be invented. The only possibility of generally-covariant Lagrangian is just (1.46) with (2.1) as the Lagrange tensor.

It would be difficult to decide at this stage what would be the physical usefulness of such models. Nevertheless, they are well-defined and they witness that the Born-Infeld scheme in many situations is the only one compatible with geometry of degrees of freedom.

But let us try to be more concrete with the problem. The question arises what might a general scheme for generally-covariant field models be. In this formulation it is too general to be effectively discussed. We know about the models of degrees of freedom admitting generally-covariant dynamical principles. Those are, among others, analytically speaking, "matrix fields", like, e.g., the metric field, i.e., the field of twice covariant tensors, the field of mixed tensors, or the field of co-frames, i.e., analytically speaking, the $n$-tuple of covector fields on the $n$-dimensional manifold ("spacetime"). In any case, in a generally-covariant field theory in $n$-dimensional "space-time" manifold, 
the field should have more than $n$ components. Because, roughly speaking, the general covariance may reduce any of $n$ field components to an arbitrarily given function form, e.g., identifying them locally with "space-time" coordinates. The simplest, academic model is that of some $N$-component scalar field in $n$-dimensional "space-time" 9, 58, 59]. As mentioned, the general covariance implies that $N>n$, otherwise any generally-covariant model will be either trivial (every field is a solution) or empty (no solutions at all).

Let the target space $V$ of dimension $N$ be endowed with some pseudo-Riemannian metric $\eta$. Obviously, the simplest situation is the one when $V$ is a linear space and $\eta \in V^{*} \otimes V^{*}$ is some "constant" (pseudo-)Euclidean metric. We may quite well assume $V$ to be a complex linear space and $h$ some sesqulinear hermitian form. However, let us focus our attention on the simplest case of a linear space with (pseudo-)Euclidean metric. Any $V$-valued scalar field $\Psi: M \rightarrow V$, i.e., analytically speaking, the multiplet of $N$ real scalar fields $\Psi^{A}$ on $M$ (some basis in $V$ fixed) induces some kind of $\Psi$-dependent metric in $M$, namely, the pull-back

$$
g[\Psi]=\Psi^{*} \cdot \eta, \quad g[\Psi]_{\mu \nu}=\eta_{A B} \Psi^{A}{ }_{\mu} \Psi^{B}{ }_{, \nu}
$$

comma, as usual, denoting the partial derivative.

Let us stress that there is no fixed metric in $M$, the space-time manifold is absolutely amorphous. The only absolute element, $\eta \in V^{*} \otimes V^{*}$, is an inhabitant of the target space $V$, it lives in "Heaven", not on "Earth". Incidentally, there are linear spaces or manifolds with intrinsic metrics, e.g., Lie algebras, Lie groups, manifolds of scalar products as discussed above, etc. So, this "absolutism" of $\eta$ need not be taken too seriously. Let us stress that in this sense our tetrad $/ n$-leg model was just completely amorphous, because nothing but the linear space structure was assumed in the target space $\mathbb{R}^{n}$ (physically $\mathbb{R}^{4}$ ). The simplest model now is the one, in which the Lagrange tensor $L_{\mu \nu}$ just coincides with $g_{\mu \nu}$. One can also consider some "potential" terms $U$, e.g., the ones built of $\|\Psi\|^{2}=\eta_{A B} \Psi^{A} \Psi^{B}$, and take

$$
L_{\mu \nu}=U(\Psi) g[\Psi]_{\mu \nu} .
$$

Of course, this is a re-definition of $\eta$ in a sense and it is essential only when $\eta$ is a constant (pseudo-Euclidean) metric in a linear space.

Euler-Lagrange equations resulting from (2.2) with $U=1$ may be invariantly written down as follows:

$$
g^{\mu \nu} \nabla_{\mu} \nabla_{\nu} \Psi^{A}=0, \quad A=1, \ldots, N,
$$

where, obviously, $g^{\mu \nu}$ are components of the contravariant inverse of $g[\Psi]$, and $\nabla_{\mu}$ are operators of the covariant differentiation in the sense of the Levi-Civita connection built of $g$. Although (2.4) formally looks like the d'Alembert equation for the multiplet $\Psi^{A}, A=1, \ldots N$, this system of differential equations is strongly nonlinear, just essentially, non-perturbatively nonlinear, because $g^{\mu \nu}$ and the Christoffel coefficients depend on $\Psi$. This dependence results in the mutual coupling of equations. For any fixed $A$, the coefficients in the equation for $\Psi^{A}$ depend on all the fields $\Psi^{B}$.

The nice invariant form (2.4) may be explicitly, but ugly, written down as follows:

$$
g^{\mu \nu} \Psi_{, \mu \nu}^{A}+\Psi_{, \nu}^{A}\left(\frac{1}{2} g^{\mu \nu} g^{\alpha \beta}-g^{\mu \alpha} g^{\nu \beta}\right) g_{\alpha \beta, \mu}=0
$$

Geometrical meaning of equations (2.3), (2.4) is that the submanifold $\Psi(M) \subset V$ is a minimal surface in the sense of pseudo-Euclidean geometry; its mean curvature does vanish [58]. This geometrical interpretation is generally true, not only in the situation when $(V, \eta)$ is flat. The system (2.4) is redundant; this is a consequence of the gauge arbitrariness corresponding to the general covariance. The Diff $M$-invariance implies that among the $N$ fields $\Psi^{A}$ there are, roughly speaking, only $(N-n)$ independent ones, while $n$ equations are superfluous and have the status of identities. There are $n$ purely gauge variables among $\Psi^{A}$ and those may be fixed by something like coordinate conditions. The simplest, although in a sense most "brutal", way of eliminating gauge 
variables is to identify some $n$-tuple of fields $\Psi^{A}$, e.g., $\Psi^{\mu}, \mu=1, \ldots, n$ (physically $n=4$ ), with space-time coordinates, i.e., to put

$$
\Psi^{\mu}=x^{\mu}, \quad \mu=1, \ldots, n .
$$

The gauge condition may be chosen in the form:

$$
\left(\frac{1}{2} g^{\mu \nu} g^{\alpha \beta}-g^{\mu \alpha} g^{\nu \beta}\right) g_{\alpha \beta, \mu}=0,
$$

quite independently of the convention (2.6); this condition is more general. This gauge condition implies that (2.5) acquires the usual "d'Alembert" form in the sense that

$$
g^{\mu \nu} \Psi_{, \mu \nu}^{A}=0 .
$$

Obviously, equation (2.7) is non-tensorial and this is correct, otherwise it would not be a coordinate condition, i.e., fixation of gauge within the Diff $M$-invariant scheme. If we assume (2.6), which is locally always correct (globally there are, obviously, some subtle problems), then our gauge equations (2.7) become identities, and they are trivially satisfied. The fields $\Psi^{a}, a=n+1, \ldots, N$, are genuine degrees of freedom.

It is convenient to choose coordinates in the target space in such a way that the matrix of $\eta$ splits into blocks:

$$
h_{\mu a}=0, \quad \mu=1, \ldots, n, \quad a=n+1, \ldots, N .
$$

Then we have

$$
g_{\mu \nu}=\eta_{\mu \nu}+\eta_{a b} \Psi_{, \mu}^{a} \Psi_{, \nu}^{b},
$$

the summation convention is meant in the sense of indices $a, b=n+1, \ldots, N$. The true dynamics, free of gauge, is described by "d'Alembert" equations for $\Psi^{a}$ :

$$
g^{\mu \nu} \Psi^{a}{ }_{, \mu \nu}=0, \quad a=n+1, \ldots, N .
$$

These are Euler-Lagrange equations for the effective Lagrangian based on the effective Lagrange tensor

$$
L(\mathrm{eff})_{\mu \nu}=\eta_{\mu \nu}+\eta_{a b} \Psi_{, \mu}^{a} \Psi_{, \nu}^{b} .
$$

The coefficients $\eta_{\mu \nu}$ play the role of something like the analytical representation of some fixed space-time metric, although, as a matter of fact, such a metric was not assumed here. Without the block-structure assumption (2.9), the effective Lagrange tensor would be given by

$$
L(\mathrm{eff})_{\mu \nu}=\eta_{\mu \nu}+2 \eta_{a(\mu} \Psi^{a}{ }_{, \nu)}+\eta_{a b} \Psi_{, \mu}^{a} \Psi_{, \nu}^{b},
$$

again with the summation convention extended over Latin indices $a=n+1, \ldots, N$.

The Born-Infeld structure, in the classical form known from electrodynamics, is easily readable here. Under the square-root sign in the effective Lagrangian we recognize the field-independent effective metric $\eta_{\mu \nu}$, the term linear in (gauge-free) field derivatives, and the term quadratic in field derivatives. The linear one is like in Born-Infeld electrodynamics, the quadratic one resembles our tetrad model (1.46), (1.47). Nevertheless, let us mention that the term quadratic in $F_{\mu \nu}$ is also possible in certain modifications of Born-Infeld electrodynamics. The corresponding Lagrange tensor might be given by something like

$$
L_{\mu \nu}=b g_{\mu \nu}+F_{\mu \nu}+d g^{\alpha \beta} F_{\mu \alpha} F_{\beta \nu}+k g^{\alpha \beta} g^{\varkappa \lambda} F_{\alpha \varkappa} F_{\beta \lambda} g_{\mu \nu},
$$

where $b, d, k$ are constants. For the fields which are not too strong, the predictions of (1.52), (2.6) are in good agreement. Another thing is that (2.6) will not certainly show any important features of the traditional model (1.52), because the latter is canonical and unique in a certain sense.

The scalar Born-Infeld models based on the effective Lagrange tensors (2.12) with $N=n+1$ (physically $n=4$ ) were used in nonlinear scalar optics, i.e., in situations where the polarization 
effects may be neglected. In any case, we have found the link between general covariance and the Born-Infeld structure of Lagrangians based on Lagrange tensors with at most quadratic dependence of field derivatives. In the scalar Born-Infeld electrodynamics, solutions appear as stationary surfaces ("minimal surfaces") of dimension four in the five-dimensional target space $V$. The corresponding metric $\eta$ has the signature $(++---)$. If we denote

$$
\left[\eta_{a b}\right]=\operatorname{diag}(\eta, 1,-1,-1,-1),
$$

then the effective Lagrangian is based on Lagrange tensor

$$
L(\mathrm{eff})_{\mu \nu}=\eta_{\mu \nu}+\eta \Psi_{, \mu} \Psi_{, \nu} .
$$

It is interesting that for the field $\Psi$ we obtain solutions of exactly the same form as the one for the scalar potential $A_{0}$ in the "usual" four-covector electrodynamics:

$$
\Psi(r)=\sqrt{\frac{A}{\eta}} \int_{0}^{r} \frac{\mathrm{d} x}{\sqrt{A+x^{4}}},
$$

where $A>0$ is some integration constant. This small fact is very interesting in itself.

To summarize, let us repeat some important special cases, which are not only suggestive but also physically interpretable.

1. $N$ is arbitrary, $n=1-$ geodetic curves.

2. $N=3, n=2$ - rubber films, soap bubbles, etc.

3. $N=4, n=1, \eta$ is Minkowskian. This is relativistic point mechanics. Obviously, for the free particle the effective Lagrangian is given by

$$
L(\mathrm{eff})=-m c^{2} \sqrt{1-\frac{v^{2}}{c^{2}}} .
$$

4. $N=4, n=2, \eta$ is Minkowskian. These are strings, 'tHooft-Polyakov-Kleinert models.

5. $N$ is arbitrary, $n=1, \eta$ is Riemannian, $U=2(E-V)$, cf. (2.3), $E$ is the fixed total energy, $V$ is potential. We easily recognize the Maupertuis variational principle.

The scalar models provide an interesting "Kunst der Fuge", the exercise for the study of essential nonlinearity in the context of general covariance. Let us stress that our $n$-leg model is in a sense "better" than all scalar Born-Infeld model, because it does not assume any target metric.

\section{Acknowledgements}

We are very grateful to our friend professor Anatoly K. Prykarpatsky for our fruitful and inspiring discussions. Some of our results were obtained within the framework of the research project 501018 32/1992 financed from the Scientific Research Support Fund in 2007-2010. We are greatly indebted to the Ministry of Science and Higher Education for this financial support. The support within the framework of Institute internal programme 203 is also greatly acknowledged. 


\section{References}

1. Bogolyubov (Jr.) N.N., Prikarpatskii A.K., Taneri U., Teoret. Mat. Fiz., 2009, 160, 249-269.

2. Eringen A.C., Nonlinear Theory of Continuous Media. McGraw-Hill Book Company, New York, 1962.

3. Eringen A.C., Mechanics of Micromorphic Continua. - In: Proceedings of the IUTAM Symposium on Mechanics of Generalized Continua, Freudenstadt and Stuttgart, 1967, E. Kröner (ed.), Vol. 18. Springer, Berlin-Heidelberg-New York, 1968, p. 18-33.

4. Liebscher D., Relativistische Theorie und Metrische Raumstruktur. - In: Gravitation and Cosmos. Beiträge zur Problem der Allgemeinen Relativitäts Theorie, R. Wahsner (Ed). Akademie-Verlag, Berlin, 1982.

5. Prykarpatsky A.K., Bogolubov (Jr.) N.N., Preprint arXiv:gr-qc/0807.3691, 2008.

6. Prykarpatsky A.K., Bogoliubov N.N., Golenia J, Taneri U, Int. J. Theor. Phys., 2008, 47, 2882.

7. Sławianowski J.J., Il Nuovo Cimento B, 1991, 106, No. 6, 645-668.

8. Sławianowski J.J., Geometrically Implied Nonlinearities in Mechanics and Field Theory. - In: Geometry, Integrability and Quantization, I. Mladenov and M. de Leon (Eds.). SOFTEX, Sofia 2007, p. $48-118$.

9. Białynicka-Birula Z., Bull. Acad. Pol. Sci., Sér. Sci. Math. Astr. Phys., 1979, 27, No. 1, 41.

10. Born M., Infeld L., Proc. Roy. Soc A. (London), 1934, 144, 425.

11. Rzewuski J., Field Theory. Part I. Classical Theory. PWN - Polish Scientific Publishers, Warsaw, 1964.

12. Dirac P., Interacting Gravitational and Spinor Fields. - In: Recent Developments in General Relativity. Pergamon Press, New York-Oxford-London-Paris, PWN - Polish Scientific Publishers, Warsaw, 1962.

13. Kobayashi S., Nomizu K., Foundations of Differential Geometry. Interscience Publishers, New York, 1963.

14. Sternberg S., Lectures on Differential Geometry. Prentice-Hall, New York, 1964.

15. Abraham R., Marsden J.E., Foundations of Mechanics (second ed.). The Benjamin-Cummings Publishing Company, London-Amsterdam-Sydney-Tokyo, 1978.

16. Arnold V.I., Mathematical Methods of Classical Mechanics. Springer Graduate Texts in Mathematics, Vol. 60. Springer-Verlag, New York, 1978.

17. Goldstein H., Classical Mechanics. Addison-Wesley, Reading, Mass., 1950.

18. Synge J.L., Classical Dynamics. Springer-Verlag, Berlin-Göttingen-Heidelberg, 1960.

19. Bogoyavlensky O.I., Methods of Qualitative Theory of Dynamical Systems in Astrophysics and Gas Dynamics. Springer, Berlin-Heidelberg-New York, 1985.

20. Marsden J.E., Ratiu T., Introduction to Mechanics and Symmetry. Springer, New York, 1994.

21. Marsden J.E., Ratiu T., Introduction to Mechanics and Symmetry. A Basic Exposition of Classical Mechanical Systems (second ed.). Springer, New York, 1999.

22. Pellegrinni C., Plebański J., Mat.-Fys. Skr. Danske Videnskab. Selsk, 1963, 2, 1-39.

23. Rubin M.B., ASME J. Appl. Mech., 1985, 52, 368-372.

24. Rubin M.B., ASME J. Appl. Mech., 1986, 53, 45-50.

25. Burov A., Chevallier D.P., On the Variational Principle of Poincare, the Poincare-Chetayev Equations and the Dynamics of Affinely Deformable Bodies, Cahier de C.E.R.M.I.C.S., 14, Mai, 1996.

26. Capriz G., Continua with Microstructure. Springer Tracts in Natural Philosophy, Vol. 35. SpringerVerlag, New York-Berlin-Heidelberg-Paris-Tokyo, 1989.

27. Capriz G., Mariano P.M., J. Elasticity, 2003, 72, 57-70.

28. Gołubowska B., Rep. Math. Phys., 2001, 48, No. 1/2, 95-102.

29. Gołubowska B., Rep. Math. Phys., 2002, 49, No. 2/3, 193-201.

30. Gołubowska B., Rep. Math. Phys., 2003, 51, No. 2/3, 205-214.

31. Gołubowska B., Infinitesimal Affinely-Rigid Bodies in Riemann Spaces. - In: Proc. of Institute of Mathematics of NAS of Ukraine, eds: A.G. Nikitin, V.M. Boyko, R.O. Popovych, and I.A. Yehorchenko, 50, Part 2, Institute of Mathematics, Kyiv, 2004, p. 774-779.

32. Gołubowska B., J. Nonlinear Math. Phy., 2004, 11, Supplement, 138-144.

33. Sławianowski J.J., Arch. Mech., 1974, 26, No. 4, 569-587.

34. Sławianowski J.J., Int. J. of Theor. Phys., 1975, 12, No. 4, 271-296.

35. Sławianowski J.J., Arch. Mech., 1975, 27, No. 1, 93-102.

36. Sławianowski J.J., Z. Angew. Math. Mech., 1982, 62, 229-240.

37. Sławianowski J.J., Analytical Mechanics of Deformable Bodies. PWN - Polish Scientific Publishers, Warszawa-Poznań, 1982 (in Polish).

38. Sławianowski J.J., Rep. Math. Phys., 1988, 26, No. 1, 73-119.

39. Sławianowski J.J., Technische Mechanik, 2002, 22, No. 1, 8-13. 
40. Sławianowski J.J., Quantum and Classical Models Based on GL( $n, \mathbb{R})$-Symmetry. - In: Proc. of the Second International Symposium on Quantum Theory and Symmetries, Kraków, Poland, July 18-21, 2001, eds: E. Kapuścik and A. Horzela. World Scientific, New Jersey-London-Singapore-Hong Kong, 2002, p. 582-588.

41. Sławianowski J.J., Rep. Math. Phys., 2003, 51, No. 2/3, 345-369.

42. Sławianowski J.J., J. Nonlinear Math. Phy., 2004, 11, Supplement, 130-137.

43. Sławianowski J.J., Classical and Quantum Collective Dynamics of Deformable Objects. Symmetry and Integrability Problems. - In: Geometry, Integrability and Quantization, I. Mladenov and A.C. Hirshfeld (Eds.). SOFTEX, Sofia 2004, p. 81-108.

44. Sławianowski J.J., Meccanica, 2005, 40, 365-387.

45. Sławianowski J.J., Kovalchuk V., Rep. Math. Phys., 2003, 51, No. 2/3, 371-379.

46. Sławianowski J.J., Kovalchuk V., Invariant Geodetic Problems on the Projective Group $\operatorname{Pr}(n, \mathbb{R})$. - In: Proc. of Institute of Mathematics of NAS of Ukraine, eds: A.G. Nikitin, V.M. Boyko, R.O. Popovych, and I.A. Yehorchenko, 50, Part 2, Kyiv, Institute of Mathematics, 2004, p. 955-960.

47. Sławianowski J.J., Kovalchuk V., J. Nonlinear Math. Phy., 2004, 11, Supplement, 157-166.

48. Sławianowski J.J., Kovalchuk V., Sławianowska A., Gołubowska B., Martens A., Rożko E.E., Zawistowski Z.J., Invariant Geodetic Systems on Lie Groups and Affine Models of Internal and Collective Degrees of Freedom, Prace IPPT - IFTR Reports, 7, 2004.

49. Sławianowski J.J., Kovalchuk V., Sławianowska A., Gołubowska B., Martens A., Rożko E.E., Zawistowski Z.J., Rep. Math. Phys., 2004, 54, No. 3, 373-427.

50. Sławianowski J.J., Kovalchuk V., Sławianowska A., Gołubowska B., Martens A., Rożko E.E., Zawistowski Z.J., Rep. Math. Phys., 2005, 55, No. 1, 1-45.

51. Sousa Dias E., A Geometric Hamiltonian Approach to the Affine Rigid Body. - In: Dynamics, Bifurcation and Symmetry. New Trends and New Tools, P. Chossat (ed.), NATO ASI Series C, Vol. 437. Kluwer Academic Publishers, Netherlands, 1994, p. 291-299.

52. Martens A., Rep. Math. Phys., 2002, 49, No. 2/3, 295-303.

53. Martens A., Rep. Math. Phys., 2003, 51, No. 2/3, 287-295.

54. Martens A., J. Nonlinear Math. Phy., 2004, 11, Supplement, 145-150.

55. Martens A., J. Nonlinear Math. Phy., 2004, 11, Supplement, 151-156.

56. Rożko E.E., Rep. Math. Phys., 2005, 56, No. 3, 311-332.

57. Binz E., Global Differential Geometric Methods in Elasticity and Hydrodynamics. - In: Differential Geometry, Group Representations and Quantization, Lecture Notes in Physics, Vol. 379, edited by J.D. Hennig, W. Lücke, and J. Tolar. Springer-Verlag, Berlin-Heidelberg, 1991.

58. Fomenko A.T., Variational Methods in Topology. Nauka, Moscow, 1982 (in Russian).

59. Ogawa N., On the Chaplygin Gas and Brane. - In: Geometry, Integrability and Quantization, I. Mladenov and M. de León (Eds.). SOFTEX, Sofia, 2007.

60. Chevallier D.P., Arch. Mech., 2004, 56, No. 4, 313-353.

61. Mariano P.M., Z. Angew. Math. Phys., 2000, 51, 752-791.

62. Möller C., Danske Videnskab. Selsk, Mat.-Fys. Meddr., 1959, 31, 14. 


\title{
Геометричні нелінійності в теорії поля, конденсованій матерії
} та аналітичній механіці

\author{
Я.Я. Славяновскі \\ Інститут фундаментальних технологічних досліджень Польської академії наук, \\ Варшава, Польща
}

У фізиці є два важливих предмети досліджень: симетрія динамічних моделей і нелінійність. Всі дійсно фундаментальні моделі $\epsilon$ інваріантними щодо певних груп симетрії. Без нелінійності також нема ні справжньої фізики, ні нашого Всесвіту, ні життя взагалі. Особливо цікавими є незбурені нелінійності, які не описуються поправками, що накладаються на деякий добре визначений бекґраунд. Ідея цієї статті є така, що існує деякий таємничий, все ще незбагненний зв'язок між суттєвою фізично важливою нелінійністю і динамічною симетрією, насамперед, великих груп симетрії. В деякому сенсі ця проблема $€$ відомою навіть в теорії солітонів, де суттєва нелінійність часто супроводжується нескінченною системою інтегралів руху - таким чином, нескінченновимірними групами симетрії. Тут ми обговорюємо загальновідомі проблеми з царини теорії поля, фізики конденсованого стану та аналітичної механіки, де зв'язок між суттєвою нелінійністю і високою симетрією є очевидним, хоча не повністю зрозумілим.

Ключові слова: електродинаміка Борна-Інфельда, конденсована матерія загальна відносність $i$ тетради, незбурена нелінійність, релятивістичний структурований континіум, динамічна симетрія 
